\title{
EFFECT OF FEMORAL NECK AXIS ANGLES ON STRESS AND STRAIN IN PROLONGED FEMUR: FE STUDY
}

\author{
Konvalinka J.*
}

\begin{abstract}
The presented study deals with the influence of the two femoral neck angles - the anteversion angle and the angle of inclination - on stress and strain in femur lengthened with the intramedullary distraction nail. Five variants of geometry model of femur were created from the CT data for the purpose of this study. All femurs were scaled to the same length. The range of angles of the specimens used in this study was $0.2^{\circ}-27.8^{\circ}$ for the angle of anteversion and $114.0^{\circ}-141.6^{\circ}$ for the inclination angle. The osteotomy and lengthening by $30 \mathrm{~mm}$ were simulated for each variant of geometry and the intramedullary distraction nail and screws were then inserted. The influence of femoral neck angles was investigated on the stress in the nail, the strain intensity in callus and the deformation along the mechanical axis. The results suggest the influence of the angle of inclination on the max. stress in nail and the influence of the anteversion angle on the max. strain intensity in callus and the deformation along the mechanical axis.
\end{abstract}

\section{Keywords: Limb lengthening, Femoral neck angles, Distraction osteogenesis, Intramedullary distraction nail, FEM.}

\section{Introduction}

To get back on one's feet can be a difficult deed, especially when you have legs of uneven length. This condition is known as leg length discrepancy and is associated with back pain, altered gait patterns or various musculoskeletal disorders such as arthritic changes or scoliosis (Gurney, 2002).

When treated surgically, the method of choice is usually limb lengthening by distraction osteogenesis (Sailhan, 2011). Distraction osteogenesis using intramedullary distraction nail (IMDN) is a modern alternative to the old Ilizarov-type external distractors that allows the patient more extensive physical activity during the treatment resulting in compressive strain in callus, which is important for the successful outcome (Schuelke, 2018). Deeper understanding of the effect of the individuality of patient's anatomy could provide means for a more effective treatment.

The aim of this study was to assess the influence of two femoral neck angles on stress and strain in femur with IMDN. Computational modeling using the finite element method was chosen for the purpose of this study.

\section{Materials and methods}

For the purpose of this study, five geometrical models of femurs were created from CT data. The geometry consisted of cortical part with medullar cavity and proximal and distal cancellous parts. All femurs were scaled to have the same length.

The femoral anteversion angle and the angle of inclination (see Fig. 1) were then measured for each of the femurs. The measurements were done according to the methodology presented in Hartel (2016) and are presented in Tab. 1.

The geometry model of retrograde IMDN with five screws was created (Paley, 2015). Retrograde IMDN (see Fig. 2a) with diameter $10.7 \mathrm{~mm}$ and length $215 \mathrm{~mm}$ was inserted into each of the five femurs, the point of entry being the intercondylar fossa. To simulate the over-reaming of the canal during the surgery, the nail insertion canal was made $2 \mathrm{~mm}$ wider than the nail diameter (Fragomen, 2017). The femurs were cut

* Ing. Jan Konvalinka: Institute of Solid Mechanics, Mechatronics and Biomechanics, Brno University of Technology; Technická 2896/2; 616 69, Brno; CZ, Jan.Konvalinka@vutbr.cz 
at the distal part of diaphysis and the distraction of $30 \mathrm{~mm}$ was simulated. The callus shape was identical in all variants with only minor differences in callus volume $(<3 \%)$.

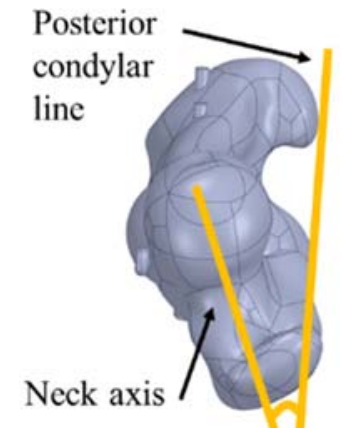

a)

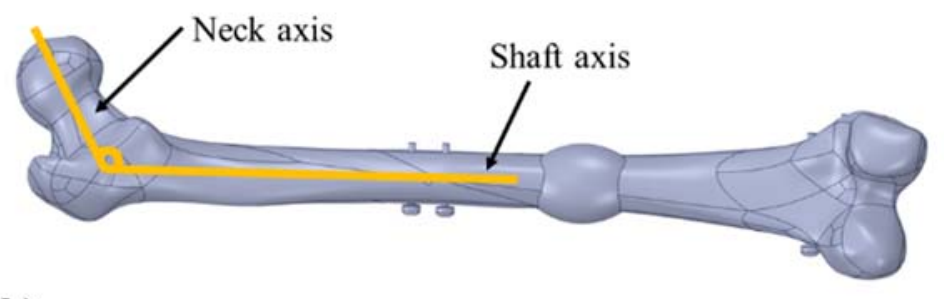

b)

Fig. 1: a) Femoral anteversion angle; b) angle of inclination.

Tab. 1: Anteversion angles and the angles of inclination of each femur.

\begin{tabular}{|c|c|c|}
\hline Femur no. & Anteversion angle $\left[{ }^{\circ}\right]$ & Angle of inclination $\left[{ }^{\circ}{ }^{\circ}\right.$ \\
\hline 1 & 26.4 & 122.5 \\
\hline 2 & 7.6 & 123.6 \\
\hline 3 & 0.2 & 141.6 \\
\hline 4 & 27.8 & 114.0 \\
\hline 5 & 3.1 & 122.3 \\
\hline
\end{tabular}

a)

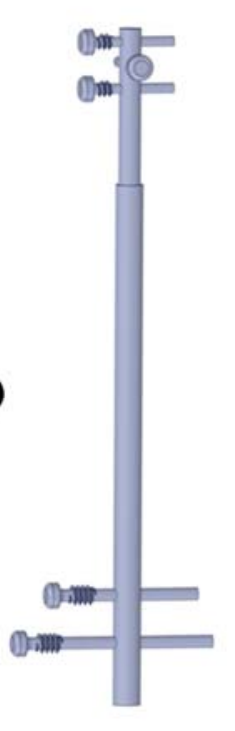

b)

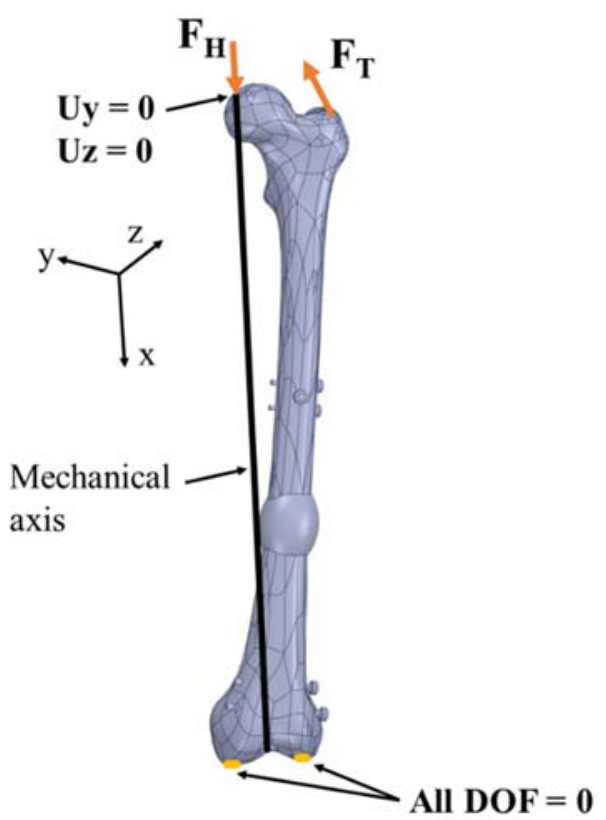

Fig. 2: a) Geometry model of IMDN; b) one variant of the complete geometry model with boundary conditions.

The computational models were solved using ANSYS 2019 software. The geometry was discretized using quadratic hexahedral and tetrahedral elements. Mesh sizing was based on a preliminary mesh sensitivity study, mesh consisted of approximately 1900000 elements (based on the variant of geometry).

Boundary conditions used in this study are depicted in Fig. 2b. Femurs were loaded with two forces - hip force $F_{H}(1000 \mathrm{~N})$ and trochanteric force $\mathrm{F}_{\mathrm{T}}(680 \mathrm{~N})$; their magnitudes were calculated according to the one-legged-stance equilibrium on lower limb. Displacements on parts of condyles were set to zero in all directions and for one point on femoral head, the non-zero displacement was allowed only in the direction of the mechanical axis (Behrens, 2009). 
Contact behaviour of bone/bone and bone/screws contact pairs was assumed to be bonded. The contact pairs of IMDN/screws and IMDN/bone were assumed to be frictional with frictional coefficients 0.1 and 0.37, respectively (López-Campos, 2018).

All material properties were assumed to be linear elastic, isotropic and homogeneous. Material properties of cortical bone were $\mathrm{E}=17 \mathrm{GPa}$ and $\mu=0.3$ and of cancellous bone $\mathrm{E}=1.3 \mathrm{GPa}$ and $\mu=0.3$ (Wang, 1998). The callus was prescribed material properties $\mathrm{E}=3 \mathrm{GPa}$ and $\mu=0.33$ (Isaksson, 2006). IMDN and screws were made from Ti6A14V with $\mathrm{E}=110 \mathrm{GPa}$ and $\mu=0.3$ (Kikuchi, 2006).

\section{Results and discussion}

For the analysis, following quantities were extracted from the results: the maximum von Mises stress in nail, the maximum deformation along the mechanical axis and the maximum strain intensity in the callus. In Fig. 3, results plotted separately against the anteversion angle and the angle of inclination are presented.

a)

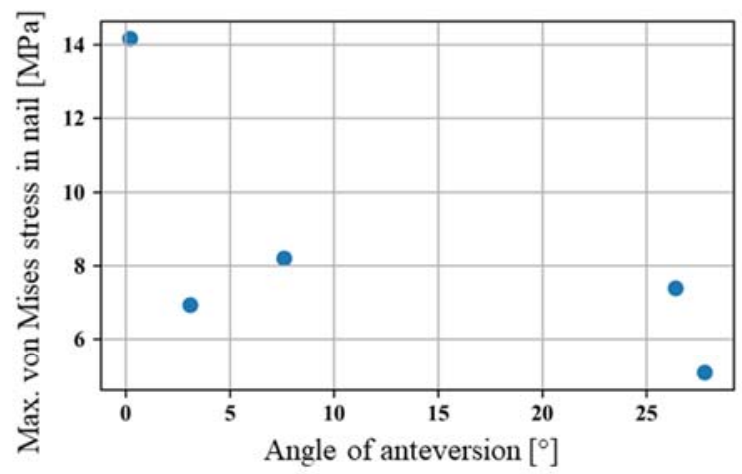

c)

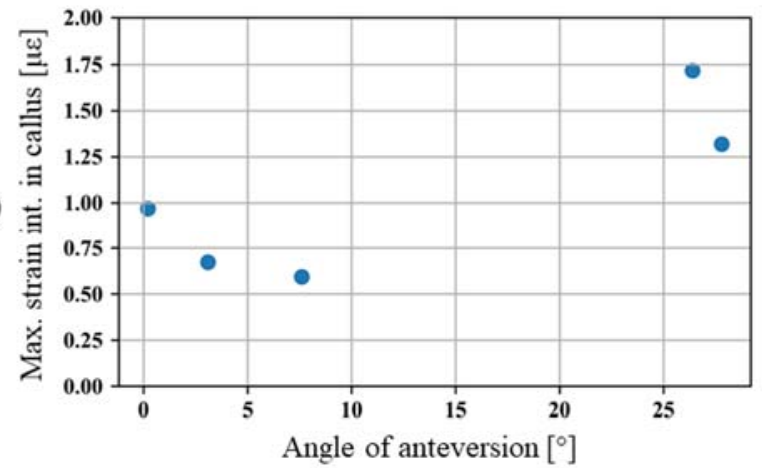

e)

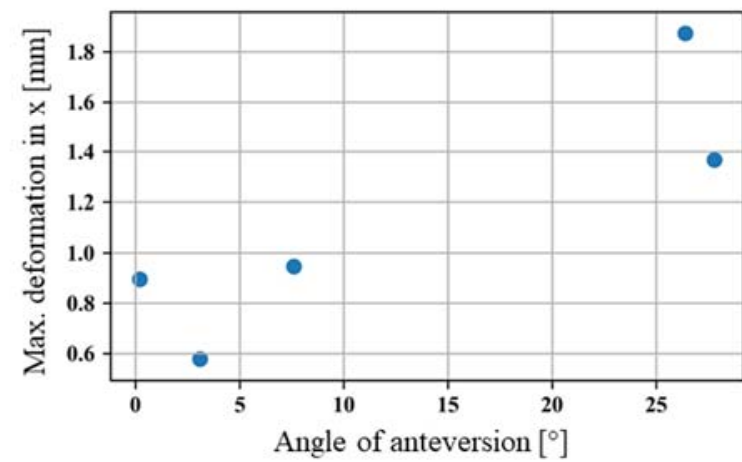

b)

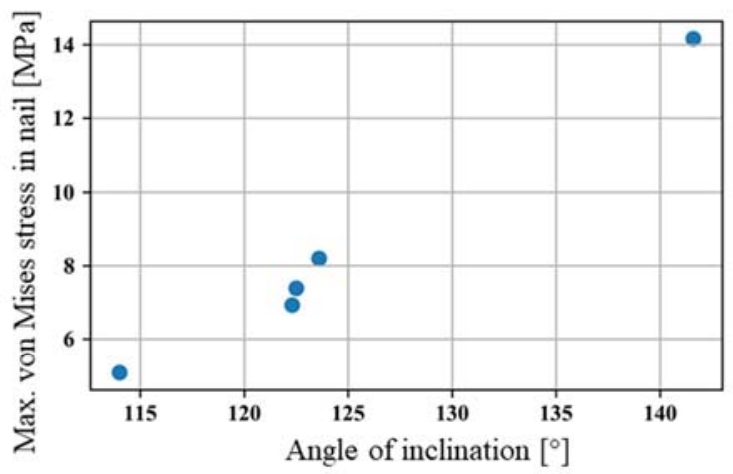

d)

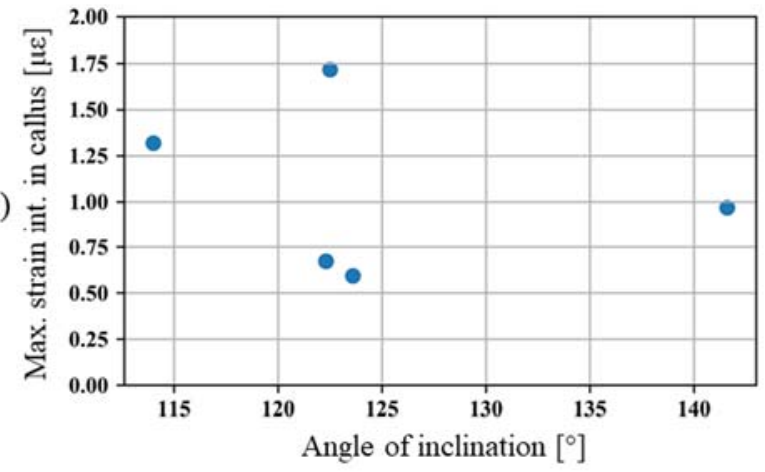

f)

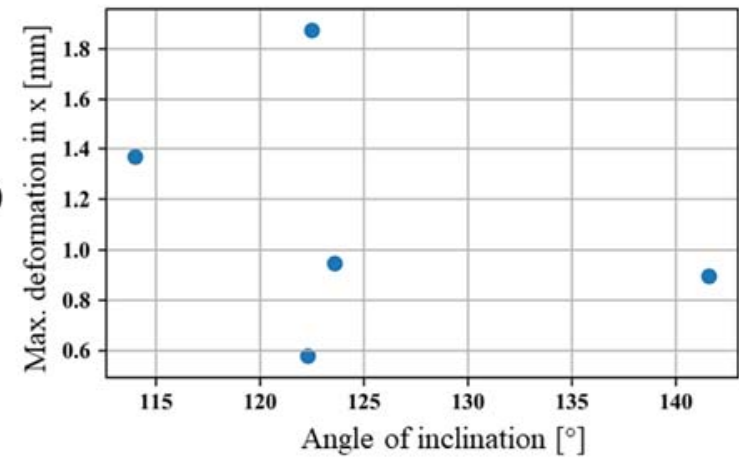

Fig. 3: Max. von Mises stress in nail vs. the anteversion angle (a) and the angle of inclination (b); max. strain intensity in callus vs. the anteversion angle (c) and the angle of inclination (d); max. def. along the mechanical axis vs. the angle of anteversion (e) and the angle of inclination (f).

Strong positive correlation ( $r=0.994, p$-value 0.001$)$ was found between the max. von Mises stress in nail and the angle of inclination (see Fig. 3b). Almost no effect of angle of anteversion was found regarding the max. von Mises stress in nail.

The variants with lower anteversion angle showed much lower max. deformation along the mechanical axis $(\mathrm{r}=0.872$, $\mathrm{p}$-value 0.054 ; see Fig. $3 \mathrm{e})$ and max. strain intensity in callus $(\mathrm{r}=0.820$, $\mathrm{p}$-value 0.089 ; see 
Fig. 3c) than the variants with higher angle of anteversion. Weak correlations were found between those two quantities and the angle of inclination.

Very different patterns of von Mises stress isolines were observed in nails (see Fig. 4), however, the maximum values of Von Mises stress were in all cases near the connection of the two nail parts.

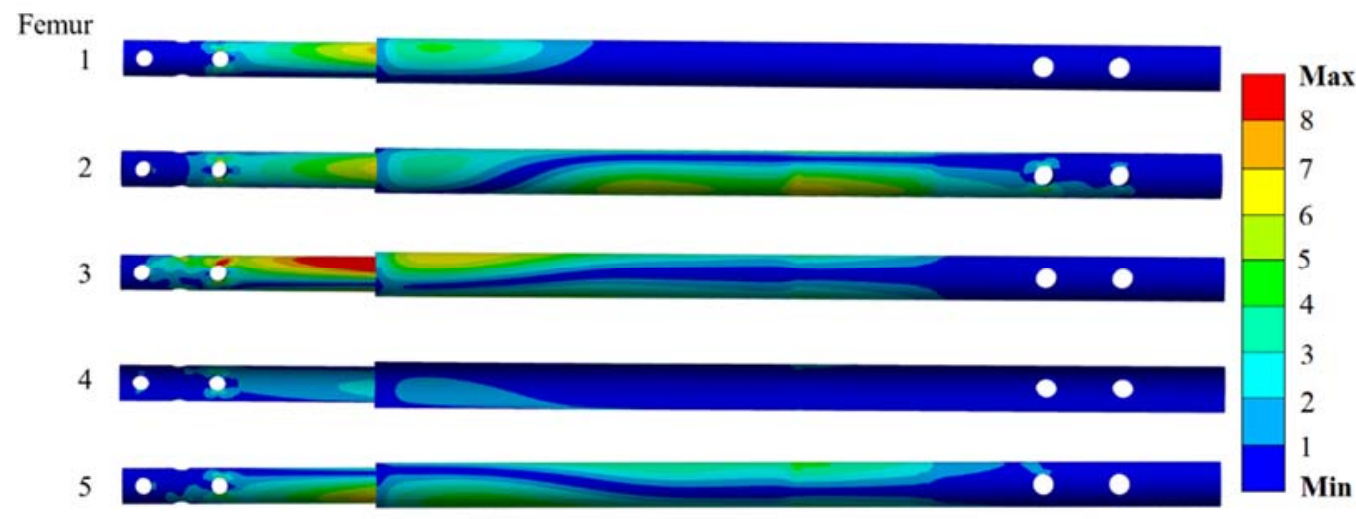

Fig. 4: Von Mises stress in nail [MPa].

\section{Conclusions}

The results suggest that the anteversion angle has a significant influence on the strain intensity in callus and on the deformation along the mechanical axis when comparing their maximum values. The angle of inclination was found to have a major influence on the maximum von Mises stress in the nail.

Despite the fact that the femoral neck angles of studied specimens are very diverse (see Tab. 1), the number of specimens used in this study was only five. If possible, further verification of presented conclusions is to be done with a higher number of different femur specimens.

\section{Acknowledgement}

This research was supported by the Faculty of Mechanical Engineering BUT grant no. FV 19-02.

\section{References}

Behrens, B., Nolte, I., Wefstaedt, P., Stukenborg-Colsman, C. and Bouguecha, A. (2009) Numerical investigations on the strain-adaptive bone remodelling in the periprosthetic femur: Influence of the boundary conditions. BioMed Eng OnLine 8, 7.

Fragomen, A. T., Rozbruch, S. R. (2017) Lengthening and deformity correction about the knee using a magnetic internal lengthening nail. SICOT-J, 3, 25.

Gurney, B. (2002) Leg length discrepancy. Gait \& Posture 15, 2, pp. 195-206.

Hartel, M. J., Petersik, A., Schmidt, A., Kendoff, D., Nüchtern, J., Rueger, J. M., Lehmann, W. and Grossterlinden, L. G. (2016) Determination of Femoral Neck Angle and Torsion Angle Utilizing a Novel Three-Dimensional Modeling and Analytical Technology Based on CT Datasets, PLoS ONE 11, 3, e0149480.

Isaksson, H., Wilson, W., van Donkelaar, C. C., Huiskes, R., Ito, K. (2006) Comparison of biophysical stimuli for mechano-regulation of tissue differentiation during fracture healing. Journal of Biomechanics, 39, 8, pp. $1507-1516$.

Kikuchi, M., Takahashi, M., Okuno, O. (2006) Elastic moduli of cast Ti-Au, Ti-Ag, and Ti-Cu alloys. Dental Materials 22, 7, pp. 641-646.

López-Campos, J.A., Segade, A., Casarejos, E., Fernández, J.R., Vilán, J.A. and Izquierdo, P. (2018) Finite Element Study of a Threaded Fastening: The Case of Surgical Screws in Bone. Symmetry, 10, 335.

Paley, D. (2015) PRECICE intramedullary limb lengthening system, Expert Review of Medical Devices, 12, 3, pp. 231-249.

Sailhan, F. (2011) Bone lengthening (distraction osteogenesis): a literature review. Osteoporosis Int 22, pp. 2011-2015.

Schuelke J, Meyers N, Reitmaier S, Klose S, Ignatius A and Claes, L. (2018) Intramembranous bone formation after callus distraction is augmented by increasing axial compressive strain. PLOS ONE 13, 4, e0195466.

Wang, C. J., Yettram, A. L., Yao, M. S., Procter, P. (1998) Finite element analysis of a Gamma nail within a fractured femur. Medical Engineering \& Physics, 20, 9, pp. 677-683. 